\title{
THE ENUMERATION OF TREE-LIKE POLYHEXES $\dagger$
}

\author{
by FRANK HARARY and RONALD C. READ
}

(Received 1st August 1968)

\section{Introduction}

A problem of considerable interest in combinatorial analysis is that of determining the number of ways in which a connected figure can be constructed in the plane by assembling $n$ regular hexagons in such a way that two hexagons abut on each other, if at all, along the whole of a common edge. Examples of these constructions can be seen in the various figures in this paper.

The nomenclature for these figures varies. They are sometimes (4) called " animals" by analogy with an organism which, starting as a single (hexagonal) cell, grows in the plane by adding further similar cells to itself. They can also be regarded as a generalization of the " polyominoes " introduced by Golomb (2). Another interpretation, of interest to the chemist, is as organic chemical compounds built up entirely from benzene rings. Thus the four simplest figures of this kind, shown in Fig. 1, represent the chemical compounds whose names are given in the figure.

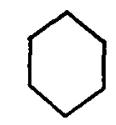

Benzene

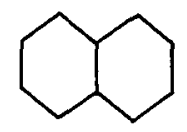

Naphthalene<smiles>C1CCC2CC3CCCCC3CC2C1</smiles>

Anthracene<smiles>C1CCC2C(C1)CCC1CCCCC12</smiles>

Phenanthrene

FIG. 1

In view of the extensive work in Balaban and Harary (1) on the listing and classification of these compounds we shall adopt their terminology and call these figures " polyhexes".

It is clear that some polyhexes will have points common to three hexagons. A polyhex for which such a point exists has been called (in (1)) peri-condensed. Another feature that a polyhex may have is that of a ring of hexagons as, for example, in the configuration consisting of a central hexagon surrounded by six adjacent hexagons. Such rings of hexagons certainly add to the difficulties of the problem of enumerating polyhexes, but since we shall shortly exclude both peri-condensed polyhexes and polyhexes having rings we need not discuss these difficulties.

† Research supported jointly by Grant 1026-66 from the Air Force Office of Scientific Research and by National Science Foundation Grant GN 2544 from the Chemical Information Program.

$$
\text { E.M.S. }-\mathbf{A}
$$


The problem of finding a formula for the total number of polyhexes with a given number $n$ of hexagons appears to be completely intractable, at least by any enumeration techniques in current use. The numbers of polyhexes for $n=1$, $2,3,4,5,6$ have been obtained by Klarner (7); they are 1, 1, 3, 7, 22, 83. Little progress has yet been made with the general problem however, the only advance in this direction known to the authors being a paper by Balaban and Harary (1) which enumerates polyhexes in which no hexagon is joined to three others.

The ends of mathematical research are often furthered by the timely adoption of the attitude epitomized in the dictum " If you can't solve the problem, change it." Adopting this attitude, not as a counsel of despair but as a realistic acknowledgment that one must learn to walk before attempting to run, we shall address ourselves to a problem of the same type as the general one mentioned above, but one in which certain simplifications have been made to bring it within the range of present enumeration techniques.

\section{The Problem}

The two sources of difficulty in the enumeration of general polyhexes are (i) the existence of a "peri-connexion", i.e., three hexagons having a common

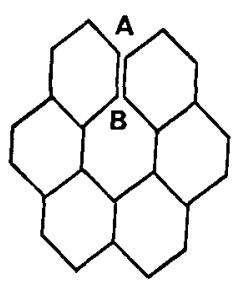

FiG. 2

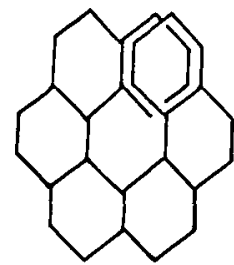

FIG. 3

point, and (ii) the existence of rings of hexagons. We easily avoid the first difficulty by stating that we shall not be interested in peri-condensed polyhexes. To avoid the second, we stipulate that we shall not allow rings of hexagons, but this stipulation requires some amplification.

It is convenient in this context to think of polyhexes as animals which have grown, by addition, from a single hexagonal cell. We shall allow an $n$-celled animal to grow into an $(n+1)$-celled animal by adding a new hexagon at an existing external edge, subject to the proviso that this does not create a point which is common to three hexagons. This may lead to a configuration like that of Fig. 2 in which a ring of six hexagons seems to be forming; but since it will not be allowed for the two edges at $A B$ to coalesce, no ring is actually formed, despite appearances. If a further hexagon is added, we may get a configuration like that of Fig. 3 where, if the hexagons are drawn accurately, two parts of the polyhex must be considered to overlap, as shown. No significance attaches to this, nor is it significant which of the overlapping parts is drawn as being on top. 
In short, then, we consider only those polyhexes which can be built up by the following recursive procedure, starting with the polyhex of one hexagon. An $n$-hex (polyhex of $n$ hexagons) is obtained from one of the $(n-1)$-hexes by adjoining a new hexagon at one of the edges on the perimeter of the $(n-1)$-hex, with the proviso that neither endpoint of the edge in question is a reentrant vertex of the perimeter of the $(n-1)$-hex (this avoids peri-connexion), and that the new hexagon and the $(n-1)$-hex have only this edge in common (this prevents the formation of rings).

Until further notice we shall regard two polyhexes as the same if one can be brought into coincidence with the other by translations and rotations in the plane. Later we shall allow reflection also, but until then a polyhex and its mirror image will in general be distinct.

By a cata-polyhex we mean a polyhex with no peri-connexion and no rings of hexagons. Consider the graph (see (11) for definitions) obtained from a given polyhex by placing a point at the centre of each hexagon and joining two such points by a line whenever the two hexagons have a common edge. Then one has a cata-polyhex if, and only if, the graph obtained in this way is a tree. It is for this reason that we can enumerate cata-polyhexes by making suitable modifications to the existing techniques for counting trees. For brevity, we use the term "polyhex" as an abbreviation for "cata-polyhex" in the rest of this paper.

\section{Polyhexes Rooted at an Edge}

We first consider polyhexes in which one peripheral edge is distinguished from the others; this is the " root edge". Since we shall later wish to attach such a polyhex by its root to another hexagon, we specify that no hexagon be attached to the root edge, or to edges next to the root edge. The enumeration of these " edge-rooted" polyhexes presents no great difficulty.

The root edge determines a unique " root hexagon" on whose boundary it lies, and we can distinguish two kinds of polyhexes, according to whether one or two hexagons are joined to the root hexagon (see Figs. $4 a$ and $4 b$ in which the root edge is drawn heavier than the others). These are the only possibilities if peri-connexion is to be avoided. Call them $S$-polyhexes and $D$-polyhexes respectively ( $S$ for single; $D$ for double).

Let $S_{n}$ and $D_{n}$ denote the numbers of $S$-polyhexes and $D$-polyhexes respectively having $n$ hexagons. Let $U_{n}$ be the total number of edge-rooted polyhexes of both kinds, so that $U_{1}=1$ and

It is easily seen that

$$
U_{n}=S_{n}+D_{n}
$$

$$
S_{n+1}=3 U_{n}
$$

since we can regard an $S$-polyhex with $n+1$ hexagons as having been constructed by adding to the root hexagon at any one of the three available edges (not the root edge or its neighbours) an $S$ - or a $D$-polyhex. 
To construct a $D$-polyhex with $n+1$ hexagons we take the root hexagon and attach edge-rooted polyhexes (either $S$ - or $D$-type) to each of the two available edges, as shown in Fig. 7b. If these polyhexes have $r$ and $s$ hexagons, then $r+s=n$ and we have

$$
D_{n+1}=\Sigma U_{r} U_{s} \quad(r+s=n, r \geqq 1, s>1)
$$

since any of the $U_{r}$ polyhexes on one side can go with any of the $U_{s}$ polyhexes on the other side.

We now define the following generating functions,

$$
S(x)=\sum_{i=1}^{\infty} S_{i} x^{i} ; D(x)=\sum_{i=1}^{\infty} D_{i} x^{i} ; U(x)=\sum_{i=1}^{\infty} U_{i} x^{i} .
$$

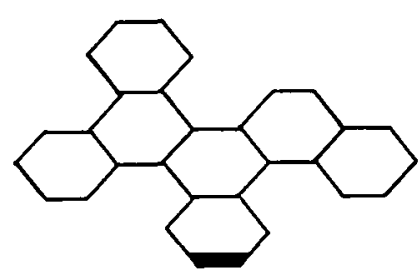

(a) An $S$-polyhex:

One hexagon adjoins the root hexagon.

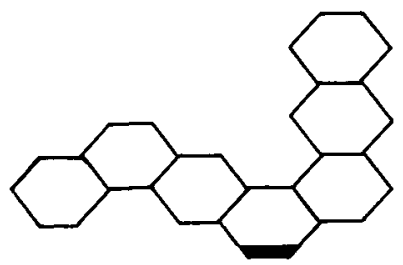

(b) A D-polyhex: Two hexagons adjoin the root hexagon.

FIG. 4

Then from (1) and the fact that $U_{1}=1, S_{1}=0$, and $D_{1}=D_{2}=0$, we have

while (2) implies

$$
U(x)=S(x)+D(x)+x,
$$

and from (3) we derive

$$
\begin{aligned}
& S(x)=3 x U(x), \\
& D(x)=x U^{2}(x) .
\end{aligned}
$$

From (5), (6) and (7) we obtain the functional equation

$$
\begin{gathered}
U(x)=3 x U(x)+x U^{2}(x)+x, \text { or } \\
x U^{2}(x)+(3 x-1) U(x)+x=0 .
\end{gathered}
$$

Solving this quadratic equation we find that

$$
U(x)=\frac{1}{2 x}\{1-3 x-\sqrt{(1-x)(1-5 x)}\}
$$

which is the required generating function. The first few terms of this series are

$$
U(x)=x+3 x^{2}+10 x^{3}+36 x^{4}+137 x^{5}+\ldots
$$

\section{Polyhexes Rooted at a Hexagon}

We now enumerate polyhexes in which one hexagon (the root hexagon) has been distinguished from the others. Any such polyhex with more than one 
hexagon can be obtained by taking the root hexagon and adjoining to one or more of its edges polyhexes rooted at an edge. There are four ways in which this can be done.

(i) only one edge-rooted polyhex is adjoined;

(ii) two edge-rooted polyhexes are joined at edges that are not diametrically opposite;

(iii) two edge-rooted polyhexes are joined at diametrically opposite edges of the root hexagon;

(iv) three edge-rooted polyhexes are adjoined.

These four possibilities are illustrated in Fig. 5, in which the root hexagon is shaded.

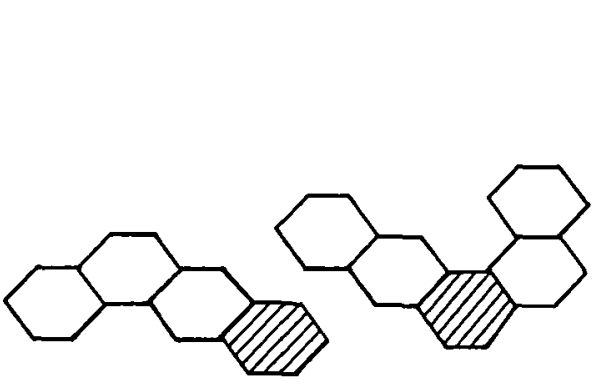

(i) (ii)

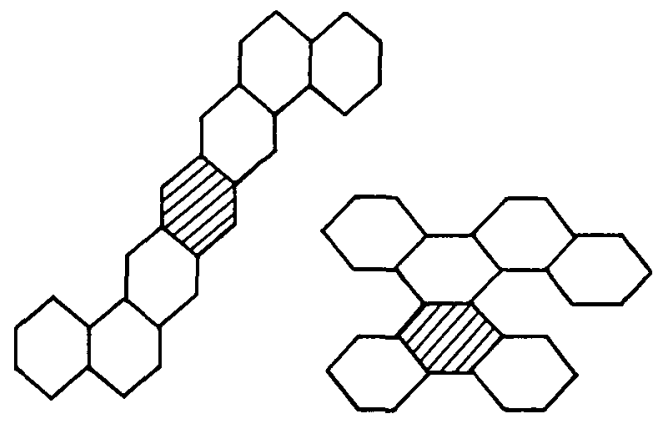

(iii)

(iv)

Fig. 5. The four types of rooted polyhexes.

We now obtain generating functions for these four types of rooted polyhexes. Clearly the number of type (i) having $n+1$ hexagons is equal to the number $U_{n}$ of edge-rooted polyhexes with $n$ hexagons. Hence the generating function for type (i) is just $x U(x)$. To find the number of type (ii), we note that since reflection in the plane is not allowed, we can distinguish between the two edges of the root hexagon at which the edge-rooted polyhexes are joined. Thus to obtain a rooted polyhex of this type having $n+1$ hexagons we choose two edge-rooted polyhexes on $r$ and $s$ hexagons where $r+s=n$. This procedure is similar to the calculation of $D_{n+1}$ given above, and we find that the generating function for the rooted polyhexes of type (ii) is $x U^{2}(x)$.

With the rooted polyhexes of type (iii) we have the possibility of polyhexes which are invariant under a rotation through $180^{\circ}$ as in Fig. 5 (iii). Such polyhexes would be counted twice by the generating function $x U^{2}(x)$. To avoid this, we make use of the well-known enumeration theorem of Pólya (9). Using the nomenclature of this paper (as described in (4) and (6) for example) we take $U(x)$ to be the figure-counting series, and the configuration group to be $S_{2}$, the symmetric group of degree 2. Applying the Pólya Theorem, we obtain

$$
Z\left(S_{2}, U(x)\right)=\frac{1}{2}\left[U^{2}(x)+U\left(x^{2}\right)\right]
$$


as the configuration counting series. This ignores the root hexagon, however, and if we include it (thus raising all the powers of $x$ by 1) we obtain

$$
\frac{x}{2}\left[U^{2}(x)+U\left(x^{2}\right)\right]
$$

as the required generating function for rooted polyhexes of type (iii).

To count rooted polyhexes of type (iv), we again use Pólya's Theorem. This time we have the possibility of symmetry under rotations through $120^{\circ}$, i.e., by elements of the cyclic group $C_{3}$ of order 3. Thus applying Pólya's Theorem and multiplying by $x$ to include the root hexagon we obtain

$$
x Z\left(C_{3}, U(x)\right)=\frac{x}{3}\left[U^{3}(x)+2 U\left(x^{3}\right)\right]
$$

as the generating function for rooted polyhexes of type (iv).

We now add these four generating functions, together with a single term of $x$ for the polyhex on one hexagon only (which otherwise is not included) to obtain the generating function $F(x)=\sum_{n=1}^{\infty} F_{n} x^{n}$ for all rooted polyhexes:

$$
F(x)=x+x U(x)+\frac{3}{2} x U^{2}(x)+\frac{1}{2} x U\left(x^{2}\right)+\frac{1}{3} x U^{3}(x)+\frac{2}{3} x U\left(x^{3}\right) .
$$

The first few terms are:

$$
F(x)=x+x^{2}+5 x^{3}+20 x^{4}+84 x^{5}+\ldots
$$

\section{Unrooted Polyhexes}

We now have to make the transition from rooted to unrooted polyhexes. The method we shall use is precisely that by which a formula for the number of trees (originally found by Otter in (8)) is most conveniently obtained from the formula for rooted trees (see (5) and (6) for the exposition of the method that we shall take as our model).

Polyhexes are essentially tree-like; in fact, as noted above, we can associate a tree with a polyhex by placing a point at the centre of each hexagon and joining by a line those pairs of points whose hexagons are contiguous. This is illustrated in Fig. 6.

Consider any unrooted polyhex, and let it have $p$ hexagons and $q$ edges that are common to two hexagons, corresponding to the $p$ points and $q$ lines of its associated tree; thus $p-q=1$. The only symmetry that a polyhex can have is by rotation in the plane through $180^{\circ}$ or $120^{\circ}$, as already remarked, since reflection is not allowed. If it has symmetry by rotation through $120^{\circ}$ then it must have a "central hexagon" which rotates into itself, and the remaining hexagons form sets of three " equivalent" hexagons which rotate into each other. If the polyhex has symmetry under a $180^{\circ}$ rotation, and has an odd number of hexagons, then it again has a central hexagon, and the remaining hexagons form a number of equivalent pairs. If the number of hexagons is 
even, however, the hexagons pair off, and the rotation takes place about the midpoint of a central edge between two hexagons (see Fig. 6 for an example of this).

For any polyhex let $p^{*}$ be the number of equivalence classes of hexagons (points of the associated tree), let $q^{*}$ be the number of equivalence classes of edges between hexagons (lines of the associated tree). Then from the above observations we easily verify the following results.

(a) If there is no symmetry, then

$$
p^{*}=p, \quad q^{*}=q .
$$

(b) If there is $120^{\circ}$ symmetry, then the central hexagon is in a class by itself and

$$
p^{*}=\frac{1}{3}(p+2), \quad q^{*}=\frac{1}{3} q .
$$

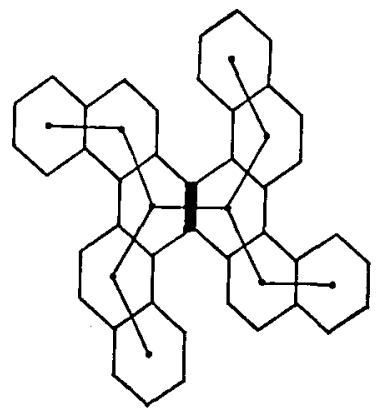

Fig. 6

(c) If there is $180^{\circ}$ symmetry and $p$ is odd, then the central hexagon is in an equivalence class by itself and

$$
p^{*}=\frac{1}{2}(p+1), q^{*}=\frac{1}{2} q .
$$

(d) If there is $180^{\circ}$ symmetry and $p$ is even, then the central edge is in a class by itself and

$$
p^{*}=\frac{1}{2} p, \quad q^{*}=\frac{1}{2}(q+1) .
$$

It follows that for any polyhex we have

$$
1=p^{*}-\left(q^{*}-s\right)
$$

where $s=1$ in case $(d)$ above, and 0 otherwise. This fundamental equation is identical in form to that for trees, cf. Otter (8).

Now sum equation (11) over all unrooted polyhexes with $n$ hexagons. The left-hand side becomes the number of unrooted polyhexes, say $h_{n}$, with reflections not permitted. The sum of the terms $p^{*}$ includes every unrooted polyhex as many times as it has equivalence classes of hexagons, which is just the number $F_{n}$ of rooted polyhexes. Similarly the summation of the terms $\left(q^{*}-s\right)$ gives the number of polyhexes in which an edge between two hexagons has been 
distinguished, except those for which there is symmetry about this edge. Let us consider the latter polyhexes first.

Polyhexes of this latter type can be constructed by joining together at their root edges two edge-rooted polyhexes of the kind enumerated in Section 2, as shown in Fig. 7. This must be done in such a way that the resulting polyhex is not symmetrical about the edge, i.e., the two edge-rooted polyhexes must not be the same. The generating function for these polyhexes can be obtained from a variation of Pólya's Theorem (see (9) or (6)) which disallows repetitions of the same figure, and is found to be

$$
\frac{1}{2}\left[U^{2}(x)-U\left(x^{2}\right)\right] \text {. }
$$

This expression can also be obtained quite easily from first principles.

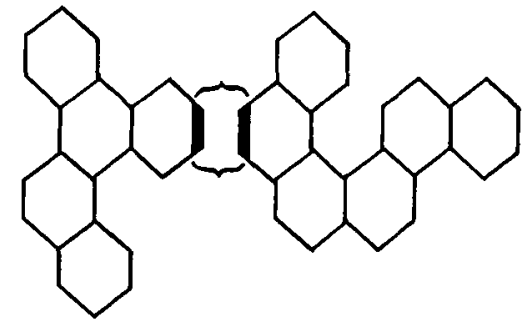

Fig. 7

Hence the summation of equation (11) over all unrooted polyhexes shows that $h_{n}$ is $F_{n}$ minus the coefficient of $x^{n}$ in $\frac{1}{2}\left[U^{2}(x)-U\left(x^{2}\right)\right]$. If $h(x)=\sum_{n=1}^{\infty} h_{n} x^{n}$ is the generating function for the numbers of unlabelled polyhexes, we have

Thus

$$
h(x)=F(x)-\frac{1}{2}\left[U^{2}(x)-U\left(x^{2}\right)\right] .
$$

$$
\begin{aligned}
h(x) & =x+x U(x)+\frac{1}{2}(3 x-1) U^{2}(x)+\frac{1}{2}(1+x) U\left(x^{2}\right)+\frac{1}{3} x U^{3}(x)+\frac{2}{3} x U\left(x^{3}\right) \\
& =x+x^{2}+2 x^{3}+7 x^{4}+18 x^{5}+\ldots
\end{aligned}
$$

This completes the enumeration of unrooted polyhexes, with no reflections allowed.

\section{Polyhexes with Reflectional Symmetry}

So far we have regarded a polyhex and its mirror image as distinct, provided that the polyhex has no symmetry which would allow it to be rotated into coincidence with its mirror image. This attitude is not a very realistic one; certainly one would not regard a plane chemical compound as having been altered by turning it over. We shall therefore now count polyhexes in a way which will not distinguish between mirror images.

The generating function $h(x)$ just obtained in (12) will in general count every polyhex (in the new sense) twice, since it includes each of a pair of mirror images. Some polyhexes will be counted only once in $h(x)$; they are those 
which have reflectional symmetry. If we can count these latter polyhexes we can deduce the required result. To do this we classify these symmetrical polyhexes into three types, as shown in Fig. 8.

It is easily seen that those of type (a) have an odd number of hexagons, while the others have an even or odd number according as $k$ is even or odd.

Let $W_{n}$ be the number of polyhexes with $n$ hexagons, having reflectional symmetry. We consider separately the cases $n$ odd and $n$ even.

Consider a polyhex for which $n$ is odd $(n=2 m+1$, say). If it is of type (b) or $(c)$ then the removal of the central hexagon results in a polyhex also of type (b) or (c). This correspondence is one-to-one, and in this way we get $W_{2 m}$ contributors to $W_{2 m+1}$. In addition we have those of type (a), and these are

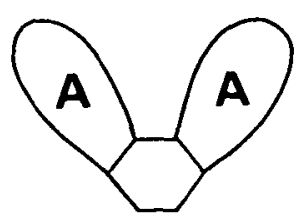

(a)

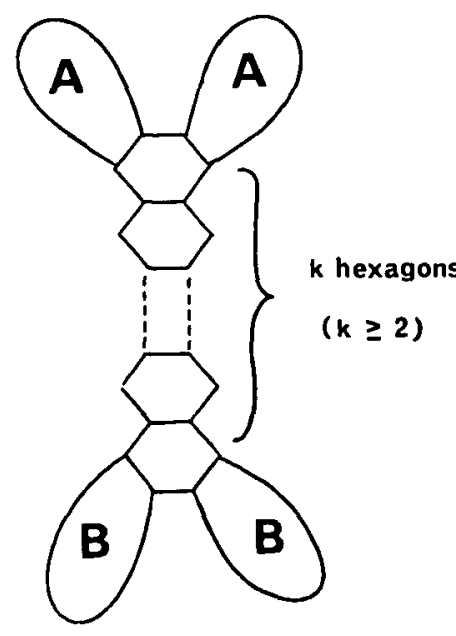

(b)

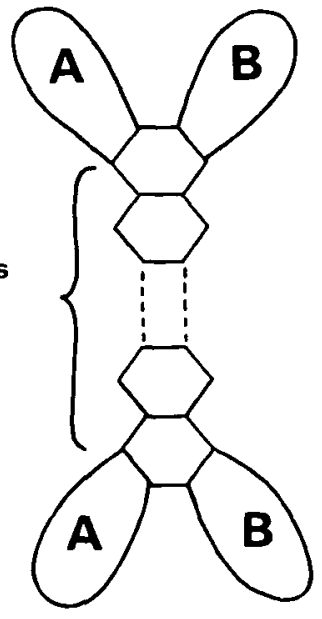

(c)

FIG. 8

specified as soon as the edge-rooted polyhex $A$ is specified. Hence there are $U_{m}$ of these. Therefore

$$
W_{2 m+1}=W_{2 m}+U_{m} \text {. }
$$

We now consider polyhexes with an even number of hexagons, say $n=2 m$. Each such hexagon will be of type $(b)$ or $(c)$. If $k>2$, then the removal of a central hexagon gives a polyhex of $2 m-1$ hexagons which is also of type (b) or (c). In addition, if the polyhex is of type (b), with $k=2$, and $B$ empty, it can be obtained from a polyhex of $2 m-1$ hexagons of type $(a)$ by adding another hexagon to the central hexagon. This accounts for all the symmetrical polyhexes of $2 m-1$ hexagons, and gives us $W_{2 m-1}$ contributors to $W_{2 m}$.

The remaining contributors to $W_{2 m}$ are

(i) those of type (c), with $k=2, A$ and $B$ being nonempty and distinct;

(ii) those of type (b), with $k=2, A$ and $B$ being nonempty and distinct; 
(iii) those of type (b) (or (c)) for which $A=B$; and

(iv) those of type (c), with $k=2$ and $B$ empty.

The polyhexes under headings (i) and (iii) can be regarded as being constructed by joining together two identical contributors to $D_{m}$, as in the derivation of equation (3). But the number $D_{m}$ will include all such polyhexes twice (because of the reflectional symmetry) except those under heading (iii), which are counted only once. Similarly (by rotating the central two hexagons through a right angle) we see that the same number $D_{m}$ includes twice all those polyhexes under heading (ii), but counts once those under (iii). Thus $2 D_{m}$ counts all of these polyhexes exactly twice, and the total number under headings (i), (ii) and (iii) is therefore $D_{m}$.

Those under heading (iv) are determined when the edge-rooted polyhex $A$ is specified. Their number is therefore $U_{m-1}$.

Taking the above results together we have

$$
\begin{aligned}
W_{2 m} & =W_{2 m-1}+D_{m}+U_{m-1} \\
& =W_{2 m-1}+U_{m}-S_{m}+U_{m-1}(\text { from (1)) } \\
& =W_{2 m-1}+U_{m}-2 U_{m-1}(\text { from (2)). }
\end{aligned}
$$

Now $W_{1}=W_{2}=1, W_{3}=2$ and $W_{4}=3$. Hence for $n=1,2$ we have

$$
W_{2 m}=U_{m} ; \quad W_{2 m+1}=2 U_{m}
$$

By mathematical induction, it follows readily from (13) and (14) that (15) is true for all $n$. Thus, if we define $W(x)=\sum_{n=1}^{\infty} W_{n} x^{n}$, we see that

$$
W(x)=x+(1+2 x) U\left(x^{2}\right) .
$$

(We are indebted to the referee for suggesting this way of deriving equation (16), instead of our much more cumbersome proof.)

\section{The Final Enumeration}

As remarked before, the generating function $h(x)$ counts every polyhex twice, except those which have reflectional symmetry, namely those that are enumerated by $W(x)$. Hence the desired polyhex generating function $H(x)=\Sigma H_{n} x^{n}$, where $H_{n}$ is the number of polyhexes on $n$ hexagons when mirror images are not regarded as distinct, is given by

$$
\begin{aligned}
H(x) & =\frac{1}{2}[h(x)+W(x)] \\
& =x+\frac{1}{2} x U(x)+\frac{1}{4}(3 x-1) U^{2}(x)+\frac{1}{4}(3+5 x) U\left(x^{2}\right)+\frac{1}{6} x U^{3}(x)+\frac{1}{3} x U\left(x^{3}\right) .
\end{aligned}
$$

By means of (8) we can express the functions $U^{2}(x)$ and $U^{3}(x)$ in terms of $U(x)$, to derive

$$
H(x)=\frac{1}{12}(1+9 x)-\frac{1}{12}(1-x)(1-5 x) U(x)+\frac{1}{4}(3+5 x) U\left(x^{2}\right)+\frac{1}{3} x U\left(x^{3}\right)
$$

which is the most convenient expression for obtaining the coefficients of $H(x)$. 
We can also use (9) to exhibit $H(x)$ as an algebraic expression in the single variable $x$.

$$
\begin{aligned}
24 x^{2} H(x)= & 12+24 x-48 x^{2}-24 x^{3}+(1-x)^{\frac{3}{3}}(1-5 x)^{\frac{3}{2}}-3(3+5 x)\left(1-x^{2}\right)^{\frac{1}{2}} \\
& \left(1-5 x^{2}\right)^{\frac{1}{2}}-4\left(1-x^{3}\right)^{\frac{1}{2}}\left(1-5 x^{3}\right)^{\frac{1}{2}} .
\end{aligned}
$$

For numerical information and as a check on the asymptotic results of the next section, the first 40 coefficients in each of the series $U(x), F(x), h(x), W(x)$, and $H(x)$ were obtained on a computer. Table 1 gives the first 12 coefficients in each of these series.

TABLE 1

\begin{tabular}{|r|rrrrrrrrrrrr|}
\hline & 1 & 2 & 3 & 4 & 5 & \multicolumn{1}{c}{6} & 7 & 8 & 9 & 10 & \multicolumn{1}{c}{11} & \multicolumn{1}{c}{12} \\
\hline$U$ & 1 & 3 & 10 & 36 & 137 & 543 & 2219 & 9285 & 39587 & 171369 & 751236 & 3328218 \\
$F$ & 1 & 1 & 5 & 20 & 84 & 354 & 1540 & 6704 & 29610 & 131745 & 591049 & 2669346 \\
$h$ & 1 & 1 & 2 & 7 & 18 & 64 & 226 & 856 & 3306 & 13248 & 53794 & 222717 \\
$W$ & 1 & 1 & 2 & 3 & 6 & 10 & 20 & 36 & 72 & 137 & 274 & 543 \\
$H$ & 1 & 1 & 2 & 5 & 12 & 37 & 123 & 446 & 1689 & 6693 & 27034 & 111630 \\
\hline
\end{tabular}

\section{Some Asymptotic Results}

It is of some interest to estimate the number of polyhexes of various kinds for large values of $n$, the number of hexagons. We first consider the number $U_{n}$ of edge-rooted polyhexes.

A theorem related to a result of Abel (see (10), p. 224) states that, if two series $\sum_{n=0}^{\infty} a_{n} x^{n}=a(x)$ and $\sum_{n=0}^{\infty} b_{n} x^{n}=b(x)$ have the same radius of convergence $R$, then

$$
\lim _{n \rightarrow \infty} \frac{a_{n}}{b_{n}}=\lim _{x \rightarrow R} \frac{a(x)}{b(x)} .
$$

Take $a(x)$ to be the function $\sqrt{(1-x)(1-5 x)}$ which appears in (9). Its radius of convergence is clearly $\frac{1}{5}$. Take $b(x)$ to be the function $(1-5 x)^{\frac{1}{2}}$, which also has this radius of convergence. Then it is easily verified that

$$
b_{n}=-\frac{1.3 \cdot 5 \ldots(2 n-3)}{n !}\left(\frac{5}{2}\right)^{n} \text {. }
$$

Hence, provided the following limit exists, we have

hence

$$
\lim _{n \rightarrow \infty} \frac{a_{n}}{b_{n}}=\lim _{x \rightarrow \frac{1}{5}} \sqrt{1-x}=\frac{2}{\sqrt{5}}
$$

$$
a_{n} \sim-\frac{1.3 \cdot 5 \ldots(2 n-3)}{n !}\left(\frac{5}{2}\right)^{n} \cdot \frac{2}{\sqrt{5}}
$$


From equation (9) we have

Consequently

$$
U_{n}=-\frac{1}{2} a_{n+1} \text { for } n>1 .
$$

$$
U_{n} \sim \frac{1.3 .5 \ldots(2 n-1)}{2(n+1) !}\left(\frac{5}{2}\right)^{n+1} \frac{2}{\sqrt{5}}=\frac{(2 n-1) !}{(n-1) !(n+1) !}\left(\frac{5}{4}\right)^{n} \sqrt{5}
$$

We now look at the numbers $H_{n}$ of polyhexes; these are given by the generating function $H(x)=\sum_{n=1}^{\infty} H_{n} x^{n}$ which was found in (18) and (19). From (18) we see that for $n>1$

$$
H_{n}=-\frac{1}{12} U_{n+1}+\frac{1}{2} U_{n-1}+\frac{3}{4} U_{n / 2}+\frac{5}{4} U_{(n-1) / 2}+\frac{1}{3} U_{(n-1) / 3},
$$

where $U_{r}$ is to be taken as zero is $r$ is not an integer. From (21) it follows readily that $U_{n / 2}, U_{(n-1) / 2}$, and $U_{(n-1) / 3}$ are small compared with the first three terms. Hence in equation (19) we can ignore everything on the right-hand side except the term $(1-x)^{\frac{3}{2}}(1-5 x)^{\frac{3}{2}}$.

Let us take

$$
f(x)=[(1-x)(1-5 x)]^{\frac{3}{2}}=\sum_{0}^{\infty} f_{n} x^{n} \text { and } g(x)=(1-5 x)^{\frac{3}{2}}=\sum_{0}^{\infty} g_{n} x^{n} .
$$

Applying (20) again, we have,

Here

$$
\begin{aligned}
\lim _{n \rightarrow \infty} \frac{f_{n}}{g_{n}} & =\lim _{x \rightarrow \frac{1}{5}} \frac{f(x)}{g(x)} \\
& =\lim _{x \rightarrow \frac{1}{4}}(1-x)^{\frac{3}{2}} \\
& =\left(\frac{4}{5}\right)^{\frac{3}{2}} .
\end{aligned}
$$

whence

$$
\sum_{n=1}^{\infty} g_{n} x^{n}=(1-5 x)^{\frac{3}{2}}
$$

$$
g_{n}=3 \cdot \frac{1 \cdot 3 \ldots(2 n-5)}{n !}\left(\frac{5}{2}\right)^{n} .
$$

From (19) we have $H_{n} \sim \frac{1}{24} f_{n+2}$, and hence

$$
\begin{aligned}
H_{n} & \sim \frac{1}{4} \cdot \frac{1.3 \ldots(2 n-1)}{(n+2) !}\left(\frac{5}{2}\right)^{n} \sqrt{5} \\
& =\frac{1}{2} \cdot \frac{(2 n-1) !}{(n-1) !(n+2) !}\left(\frac{5}{4}\right)^{n} \sqrt{5} .
\end{aligned}
$$

A consequence of (22) and (23) is that

$$
\frac{U_{n}}{(n+2) H_{n}} \sim 2 \text {. }
$$


For $n=40$ we have a verification of (24); for

and thus

$$
\begin{aligned}
& U_{40}=21949989502339625620014855 \\
& H_{40}=256364771375268976315575
\end{aligned}
$$

$$
\frac{U_{40}}{42 H_{40}}=2.038574935 \ldots
$$

\section{REFERENCES}

(1) A. T. Balaban and F. Harary, Chemical graphs. V. Enumeration and proposed nomenclature of benzenoid cata-condensed polycyclic aromatic hydrocarbons, Tetrahedron 24 (1968), 2505-2516.

(2) W. S. Golomb, Polyominoes (Scribner's, New York, 1965).

(3) F. Harary, The number of linear, directed, rooted and connected graphs, Trans. Amer. Math. Soc. 78 (1955), 445-463.

(4) F. HARARY, Graphical enumeration problems, Chapter 1 in Graph Theory and Theoretical Physics (F. Harary, ed.) (Academic Press, London, 1967, pp. 1-41).

(5) F. HARARY and R. Z. NoRman, Dissimilarity characteristic theorems for graphs, Proc. Amer. Math. Soc. 11 (1960), 332-334.

(6) F. HARARY and G. PrIns, The number of homeomorphically irreducible trees and other species, Acta Math. 101 (1959), 141-162.

(7) D. KLARNER, Some results concerning polyominoes, Fibonacci Quarterly 3 (1965), 9-20.

(8) R. OTTER, The number of trees, Ann. of Math. (2) 49 (1948), 583-599.

(9) G. Pólya, Kombinatorische Anzahlbestimmungen für Gruppen, Graphen und chemische Verbindungen, Acta Math. 68 (1937), 145-354.

(10) E. C. TItchMarsh, Theory of Functions, 2nd edition (Oxford University Press, 1939).

(11) F. Harary, Graph Theory (Addison-Wesley, Reading, Mass., 1969).

UNIVERSITY OF MICHIGAN

ANN ARBOR, U.S.A.

AND

UNIVERSITY OF THE WEST INDIES

KINGSTON, JAMAica 